\title{
Sonja Scheffler, Das Bild des Unternehmers im Spiegel der Gewerbepolitik im nachnapoleonischen Preußen
}

Frankfurt am Main, Berlin, Bern, New York, Oxford, Wien : Peter Lang (Volkswirtschaftliche Analysen, 15), 2009, 268 p., 63,95€

Guillaume Garner

\section{CpenEdition}

\section{Journals}

Édition électronique

URL : http://journals.openedition.org/ifha/7716

DOI : 10.4000/ifha. 7716

ISSN : 2198-8943

Éditeur

IFRA - Institut franco-allemand (sciences historiques et sociales)

Référence électronique

Guillaume Garner, «Sonja Scheffler, Das Bild des Unternehmers im Spiegel der Gewerbepolitik im nachnapoleonischen Preußen », Revue de l'IFHA [En ligne], Date de recension, mis en ligne le 15 décembre 2013, consulté le 22 septembre 2020. URL : http://journals.openedition.org/ifha/7716 DOl : https://doi.org/10.4000/ifha.7716

Ce document a été généré automatiquement le 22 septembre 2020.

(CIFHA 


\section{Sonja Scheffler, Das Bild des Unternehmers im Spiegel der Gewerbepolitik im nachnapoleonischen Preußen}

Frankfurt am Main, Berlin, Bern, New York, Oxford, Wien : Peter Lang

(Volkswirtschaftliche Analysen, 15), 2009, 268 p., 63,95€

\section{Guillaume Garner}

Tiré d'une thèse de science économique, cet ouvrage se propose de retracer l'évolution de l'image de l'entrepreneur dans la théorie économique et dans la politique économique entre les années 1740 et le milieu du XIXe siècle, à partir essentiellement du cas des États prussiens. Si le choix de lier théorie et pratique politique tout comme le découpage chronologique choisi semblent prometteurs, l'ouvrage se révèle décevant à la lecture.

En premier lieu, la sélection des auteurs étudiés dans la partie «théorique » de cette étude n'est pas justifiée et reprend largement la liste des "grands noms » fournie par nombre de manuels d'histoire de la pensée économique: Cantillon, Quesnay, Turgot sont ainsi étudiés mais le lecteur n'apprend rien de la réception en Prusse de «l'image de l'entrepreneur » élaborée par ces auteurs (bien que le chapitre en question soit intitulé «les fondements théoriques de la politique industrielle prussienne»). Adam Smith est également et sans surprise l'objet d'un long chapitre, qui est suivi d'une étude de sa réception en Allemagne, et c'est ici qu'apparait le second défaut majeur de ce livre: il repose sur une bibliographie à la fois datée et surtout partielle. L'auteur affirme ainsi (p. 179) que les caméralistes ont à peine reçu la Richesse des Nations, conclusion que les travaux de Marcus Sandl ont réfutée. Mais celui-ci n'est pas cité, de même que l'étude d'Axel Rüdiger sur l'université de Halle et notamment sur Christian Daniel Voss. L'ouvrage toujours fondamental de Keith Tribe est cité avec économie et n'est en tout cas pas exploité. Les chapitres portant sur la politique économique menée en Prusse sont plus convaincants car plus directement centrés sur ce qui est le sujet de 
cet ouvrage. Mais le lecteur s'étonne de n'y trouver mentionnés ni l'ouvrage de Reinhart Koselleck sur les réformes prussiennes, ni l'étude pourtant classique de Barbara Vogel sur les réformes économiques du chancelier Hardenberg. Face à cette accumulation de lacunes, faut-il en encore s'étonner que l'auteur se soit dispensé d'étudier les écrits de Johann Gottfried Hoffmann, qui fut à la fois professeur d'économie politique à Königsberg et directeur du département de l'industrie et du second Bureau de statistique prussien? Mais il est vrai que celui-ci n'est pas entré dans la liste canonique des auteurs à connaître, à la différence de Sartorius, Kraus et Jakob qui sont, eux, étudiés. Où l'on voit que le conservatisme et le poids de la tradition ne caractérisent pas seulement les corporations artisanales du début du XIXe siècle...

Ces lacunes sont d'autant plus dommageables que cet ouvrage repose en grande partie sur de la littérature secondaire et que l'étude des sources y occupe une place relativement faible. Abondamment citée, cette historiographie n'est pas critiquée. La légende de l'entrepreneur de Prusse occidentale en butte à l'interventionnisme de l'État mercantiliste est ainsi reprise (p. 36) à propos des « frères Leyen » (von der Leyen en réalité), bien que Peter Kriedte ait souligné comment ces industriels de Krefeld se sont imposés en grande partie grâce au soutien de l'administration prussienne.

Écrit dans un style qui se caractérise par de très nombreuses répétitions, cet ouvrage est décevant malgré l'intérêt des thématiques abordées.

\title{
INDEX
}

Thèmes : Wirtschaftsgeschichte

Index chronologique : Epochenübergreifend

\author{
AUTEUR \\ GUILLAUME GARNER
}

ENS Lyon/ UMR 5190 LARHRA 